\title{
BRAF-Inhibitor auch langfristig effektiv
}

Etwa $50 \%$ der malignen Melanome weisen aktivierende Mutationen (V600Mutationen) in der Serin-Threonin-Proteinkinase BRAF auf. Was kann der orale BRAF-Inhibitor Vemurafenib bei diesen Patienten leisten?

atienten mit metastasiertem Melanom hatten bisher nicht viele therapeutische Optionen. Im Jahr 2002 wurde entdeckt, dass etwa die Hälfte der Melanome eine Mutation in der Serin-Threonin-Proteinkinase BRAF aufweist, die die Zellproliferation begünstigt und die Apoptose hemmt. Der Kinase-Inhibitor Vemurafenib wirkt spezifisch auf Zellen mit BRAF-V600-Mutation und verbesserte in einer Phase-III-Studie das Gesamtüberleben gegenüber Dacarbazin bei bisher unbehandelten Patienten mit BRAF-V600-Mutation. Die mediane Beobachtungsdauer beträg allerdings bisher nur etwas weniger als vier Monate.
In einer multizentrischen Phase-IIStudie wurde Vemurafenib (960 mg, zweimal täglich) über median 12,9 Monate bei 132 Patienten mit vorbehandeltem metastasiertem Melanom mit BRAF-V600Mutation untersucht. Die Gesamtansprechrate betrug $53 \%$, wobei $6 \%$ der Patienten vollständig und $47 \%$ partiell ansprachen. Median betrug die Dauer des Ansprechens 6,7 Monate, das progressionsfreie Überleben 6,8 Monate. Eine primäre Progression war nur bei $14 \%$ der Patienten zu beobachten. Das mediane Gesamtüberleben lag bei 15,9 Monaten.

Die häufigsten unterwünschten Ereignisse waren Arthralgien, Hautausschlag,
Fotosensitivität, Fatigue und Alopezie. Bei einigen Patienten stiegen die Leberenzyme vorübergehend an. Vier Patienten brachen die Therapie wegen unerwünschter Ereignisse $\mathrm{ab}$, einer starb nach einer schnellen Progression und akutem Nierenversagen, das möglicherweise auf die Studienmedikation zurückging. $26 \%$ der Patienten entwickelten kutane Plattenepithelkarzinome oder Keratoakanthome.

Fazit: Mehr als die Hälfte der Patienten mit vorbehandelten metastasierten Melanomen mit BRAF-V600-Mutation sprach auf den BRAF-Inhibitor an. Die lange Beobachtungsdauer in dieser Studie lässt auf ein medianes Gesamtüberleben von fast 16 Monaten schließen. JudithNeumaier

Sosman JA et al. Survival in BRAF V600-mutant advanced melanoma treated with vemurafenib. N Engl J Med. 2012;366(8):707-14.

\section{Verhaltenstherapie dämpft Hitzewallungen}

Nächtliche Schweißattacken und lästige Hitzewallungen - die meisten Frauen nach einer Brustkrebstherapie kennen dieses Problem. Britische Forscher testeten, ob eine kognitive Verhaltenstherapie die Beschwerden lindern kann.

$\mathrm{D}$ as Team vom Institut für Psychiatrie des King's College in London nahm in ihre Studie knapp 100 Frauen auf, die unter mindestens zehn problematischen Hitzewallungen oder nächtlichen Schwitzattacken pro Woche litten. Alle hatten eine Brustkrebstherapie hinter sich und keine Hinweise auf weitere Läsionen.

Die Hälfte erhielt sechs Wochen lang je 90 Minuten pro Woche eine kognitive Verhaltenstherapie. Darin erfuhren sie, welche Faktoren die Menopausebeschwerden verstärken können (Stress, übertriebene Sorgen aufgrund der Symptome, ungünstige Schlafgewohnheiten). Die Psychologen arbeiteten mit ihnen Strategien aus, um solche Verstärker zu vermeiden, und trainierten Atem- und Entspannungsübungen bei Hitzewallungen. Schließlich erhielten die Frauen eine CD mit Anleitungen für das Training zu Hause. Die andere Hälfte bekam eine Standardnachsorge mit kurzen Informationen zu Optionen gegen Hitzewallungen.

Nach neun und 26 Wochen wurden die Frauen nach Häufigkeit und Stärke der

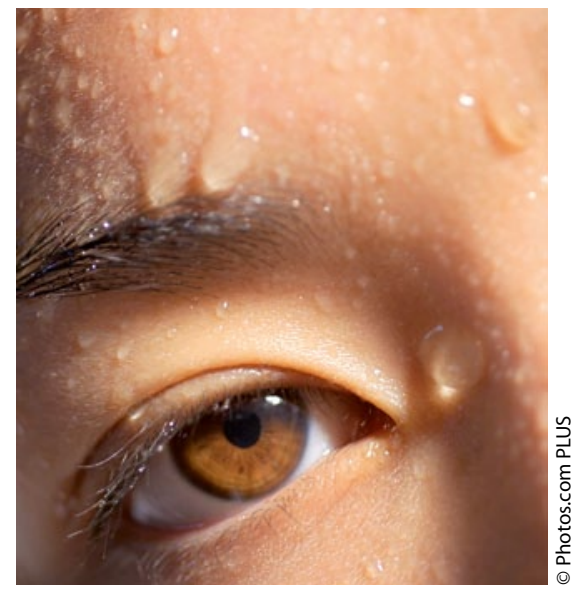

Wenn nachts plötzlich der Schweiß ausbricht, kann es helfen, Stress abzubauen oder Schlafgewohnheiten zu ändern.

Hitzebeschwerden befragt und die Ergebnisse auf einer zehnstufigen Skala dargestellt, bei der ein Unterschied von zwei Punkten als klinisch relevant gilt.

$\mathrm{Zu}$ Beginn der Studie lagen die Werte bei 6,5 (Verhaltenstherapie) und 6,1 Punkten (Kontrollgruppe). Nach sechs
Wochen Verhaltenstherapie war der Wert deutlich stärker zurückgegangen als in der Kontrollgruppe (um 3,1 vs. 1,1 Punkte). Insgesamt gingen die Beschwerden in der Gruppe mit Verhaltenstherapie um 46\% zurück, mit Standardnachsorge nur um $19 \%$. Die Unterschiede waren auch nach 26 Wochen, also mehrere Monate nach Therapieende, noch ähnlich groß: So waren die Menopausebeschwerden mit Verhaltenstherapie um $52 \%$, ohne nur um $25 \%$ zurückgegangen. Eine klinisch signifikante Reduktion der Beschwerden um zwei Punkte gelang mit Verhaltenstherapie nach neun Wochen (65 versus $38 \%$ ) und nach 26 Wochen (78 versus $33 \%$ ) ebenfalls deutlich häufiger. Zudem verbesserten sich mit Verhaltenstherapie auch Schlaf, Stimmung und Lebensqualität deutlich stärker.

Fazit: Die Forscher schließen daraus, dass eine kognitive Verhaltenstherapie bei Frauen nach einer Brustkrebsbehandlung durchaus helfen kann, typische Menopause-Symptome zu lindern. Thomas Müller

Mann E et al. Cognitive behavioural treatment for women who have menopausal symptoms after breast cancer treatment (MENOS 1): a randomised controlled trial. Lancet Oncol. 2012;13(3):309-18. 